\title{
Enhancement of Assamese Speech Signals Using Learning Based Techniques
}

\author{
Mridusmita Sharma* and Kandarpa Kumar Sarma \\ Department of Electronics and Communication Engineering, \\ Gauhati Univeristy, Guwahati, Assam, India
}

\begin{abstract}
For efficient recognition and proper interpretation of a speech signal in ASR models, de-noising of corrupted signals becomes very important. There are many traditional noise removing techniques which proved to be reliable but researchers have found the learning based approaches to be more fruitful than those traditional methods. Deep learning techniques have been popularly used in speech de-noising and recognition purposes and have become an integral part of such ASR systems. In this work, Auto-encoders and other ANN models have been implemented to remove noise from Assamese speech signals irrespective of the speaker, gender and the dialectal identity of the samples. The sample set consist of clean Assamese sentences of two, three, four, five and six words and corrupted signals with AWGN variation of $3 \mathrm{~dB}$ to $-3 \mathrm{~dB}$ which increases the sample size and also makes the system robust. Despite of certain limitations, the satisfactory experimental results justifies the proposed de-noising method for Assamese speech.
\end{abstract}

KEY WORDS: AUTO-ENCODER; ASSAMESE; ARTIFICIAL NEURAL NETWORK; NOISE ; SPEECH.

\section{INTRODUCTION}

The presence of noise signal can be felt almost everywhere. The desired output of all the applications that take voice or speech signals as input is generally corrupted by noise that is present at the surrounding or in the transmission channel. De-noising is an important operation which is necessary for determining the efficient outcome of the speech signals (Upadhyay et. al, 2013). Different techniques performing speech signal de-noising have been reported by various researchers over the years for various speech recognition applications. Despite of all the available techniques, the requirement of an automatic speech de-noising method have laid the stress

Biosc Biotech Res Comm P-ISSN: 0974-6455 E-ISSN: 2321-4007

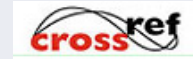

Identifiers and Pagination

Year: 2021 Vol: 14 No (5) Special Issue

Pages: 100-104

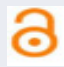
Article Information Received: $11^{\text {th }}$ Jan 2021 Accepted after revision: $28^{\text {th }}$ Mar 2021

This is an open access article under Creative

Commons License Attribn 4.0 Intl (CC-BY). DOI: $h$ ttp://dx.doi.org/10.21786/bbrc/14.5/20 on designing a learning aided approach. Learning based technique such as Shallow Neural Network (SNN) and deep learning such as Auto-encoder (AE) have proved to be efficient for implementation as a non-linear filter for noise removal (Tamura et al., 1989, Osaka et al., 2015 Et Badri, 2010).

The non-linear nature of Artificial Neural Network (ANN) and its ability to retain its learning from the environment in supervised and/or unsupervised manner makes them highly suitable for noise removal applications (Wan et al., 1999). It has been reported by various researchers over the past decade that learning based techniques have been extensively used for the purpose of speech signal denoising applications. In (Cox, 1988), it has been reported that ANN can act as a filter in noise reduction application. Several distinct neural network based frameworks have been reported in the literature where the ability of the learning based technique can be implemented in various fields of application (Feizi, 2019). In (Rajini et al., 2019), the authors have concluded that the Adaptive filter using LMS filter technique is the most suitable method for speech signal de-noising. They have also mentioned that among the neural network method ADALINE technique

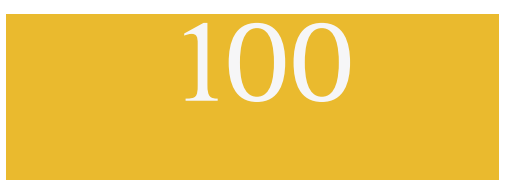


performs better, but the main limitation of the ANN method is the training latency.

In this paper we have reported a Stacked Auto-encoder based speech signal de-noising approach where the results are compared with various other learning based classifiers such as Recurrent Neural Network (RNN), Feed Forward Neural Network (FFNN) for the purpose of enhancement of the signal by obtaining lower Mean Square Error (MSE) and higher Peak Signal to Noise Ratio (PSNR) value. The rest of the paper is organized in the following sections. Section 2 includes certain basic considerations and related literature. Section 3 describes the proposed work. Experimental results are highlighted in Section 4. Section 5 concludes the paper.

Basic Considerations: This section provides a brief description of certain relevant topics related to the design of a speech signal de-noising system:

Denoising: With the rapid increase of speech based communication applications, the need to reduce the noise from the speech samples have become an important requirement. The main aim of the speech denoising model is to remove the noise from the speech signals while enhancing the speech quality and also the intelligibility of the speech signal. Many researchers have proposed a host of techniques which can be implemented for reduction of noise in speech signals (Osaka et al., 2015 Et Daqrouq et al., 2009), but the current work focuses in the implementation of ANN based techniques for noise removal in Assamese speech.

Feed Forward Neural Network (FFNN): Figure 1. shows the architecture of a multilayer FFNN. The neurons in all the layers except the output layer of a FFNN architecture has a forward connection to the units in the subsequent layer. The connections have weights associated with them and the nonlinear transfer functions of the neurons allows the network to learn the linear and non-linear relationship between the input and output samples. The learning takes place with the back-propagation technique (Svozil et al., 1997).

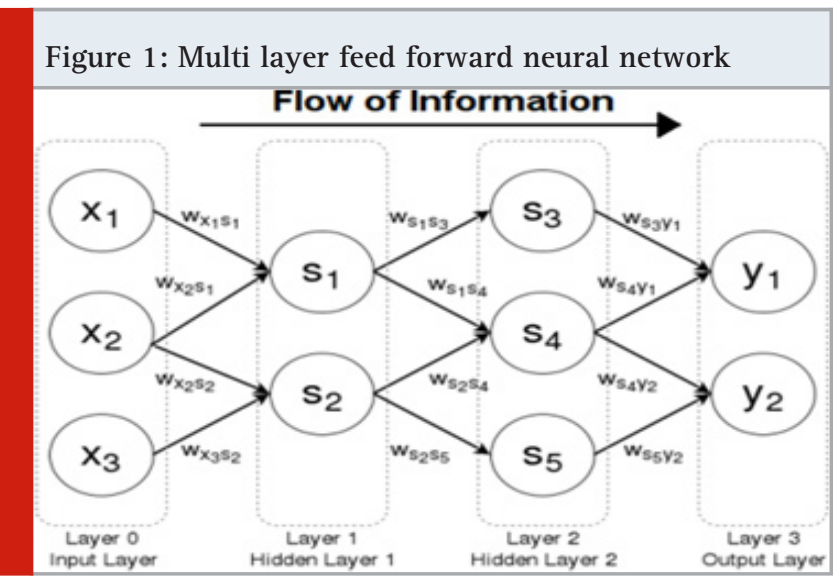

Recurrent Neural Network: ANN has the ability to learn dynamic and time series relations between the input and the output. This is because of the network architecture where the output depends not only in the current input but also on the previous state of the network (Mikolov et al., 2010). The feedback loop present in the RNN architecture help the network retain the temporal information present in the samples. Figure 2. shows an RNN architecture.

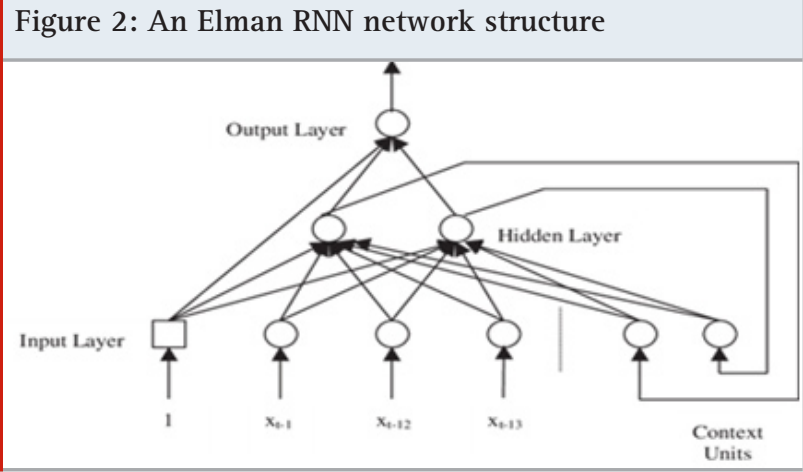

Auto-Encoder: Auto-encoder which is a form of feed forward neural network, learns by unsupervised methods. The main objective of an Auto-encoder is to learn a representation by reducing the dimension of the sample. The auto-encoder has the ability to learn the sample representation by ignoring the noise present in it and therefore act as a de-noising filter. Schematic of a basic AE is shown in Figure 3.

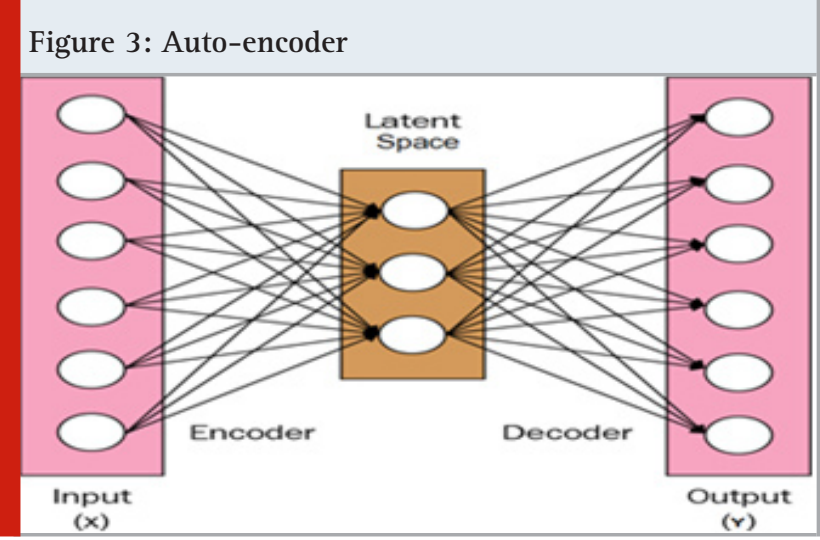

The AE has three segments which are the encoder, bottleneck part and the decoder. The low dimensional representation of the input samples takes place in the bottleneck part which is also known as the latent space representation. The input sample in the encoder part can be represented as $h=f(x)$, where is the input sample set and the decoder part can be mathematically represented as $o=g(h)$ where o is the similar representation of the input space $x$.

Stacked Autoencoders (SAE) as shown in Figure 4 are auto-encoders stacked together to form a multi layer network which has the characteristics of performing noise reduction and is found to be resilient to all types of background noise variations. More number of autoencoders stacked together give a better representation of the samples. SAE being an unsupervised method is useful for situations where there are noises (Baldi, 2012). 
In our work we have stacked two auto-encoders, the output equation of which can be shown in Equation (1) (Sharma et al., 2019).

$$
\sum_{r=1}^{Q} X_{E r}=f_{r}\left(\sum_{r=1}^{Q}\left(\sum_{l=1}^{P} X_{D l} \cdot w_{5 l r}+e_{1 r}\right)\right)
$$

where $r=1,2, \ldots Q, Q$, is the size of the output layer of the second auto-encoder.

Figure 4: Generic Stacked Auto-encoder

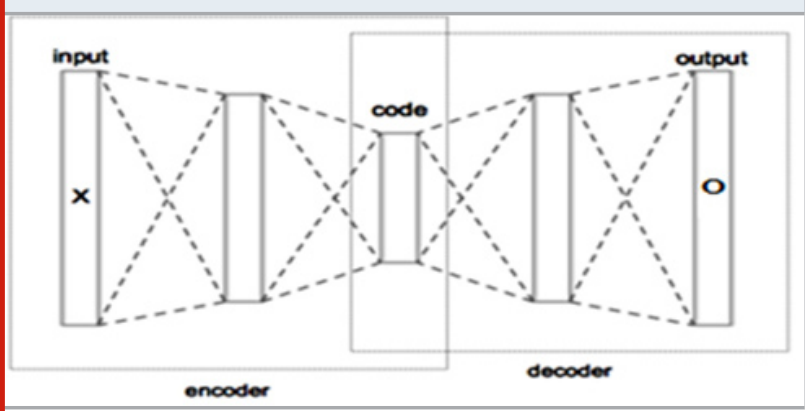

Proposed Work: The basic block diagram of our proposed work is shown in Figure. 5. Here, different learning based models are implemented to remove the noise from the sample signals and obtain satisfactory RMSE and PSNR values. The learning based models are trained extensively and subsequently tested to obtained the enhanced signal.

Dataset: For the automatic de-noising of speech samples, we have considered the Assamese language which is ethnographically rich and diverse. The samples consists of telephonic speech from 12 speakers. Each speaker uttered different Assamese sentences for at least three utterances. Further, the speech samples were subjected to SNR variations by adding AWGN noise between 3 $\mathrm{dB}$ to $-3 \mathrm{~dB}$ which increases the dataset and makes the system robust. Thus a set of 1260 speech samples were collected.

\section{METHODOLOGY}

In this work, the basic approach is based on the data collection for training and testing of the learning based classifiers for the denoising purpose. The configuration of the network is an important task during the process. The performance measure of the network models are done by considering the RMSE and PSNR values. The model learns in a supervised manner through the process of obtaining a 'mapping' between a clean and noisy speech spectra, with root mean squared error (RMSE) loss function shown in Equation (2) which is a way to observe how well the model has fitted to the training data.

$$
R M S E=\sqrt{\frac{1}{n} \sum_{k}\left(\hat{y}_{k}-y_{k}\right)^{2}}
$$

where, and are the estimated observation and the actual observation respectively.
Figure 5: Work flow of the proposed system

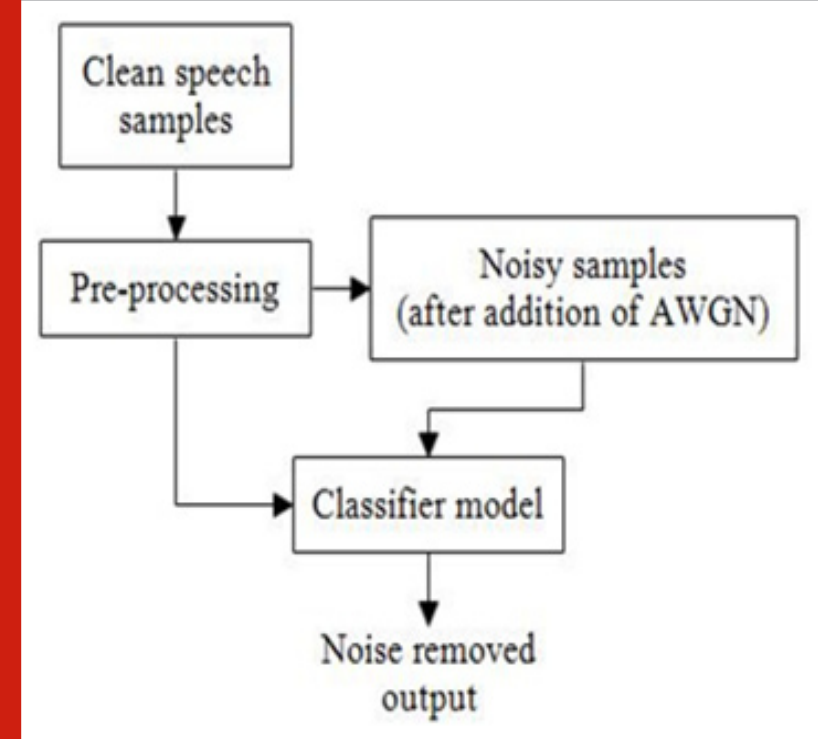

PSNR will be calculated between the output clean signal and input noisy signal to verify the quality of signal enhancement system. The efficiency of this system was tested by several speech signals.

\section{RESULTS AND DISCUSSION}

As mentioned earlier, the goal of our work is to perform the speech de-noising using learning based classifiers. RMSE and PSNR values were considered for the performance evaluation of the proposed system. High value of PSNR and lesser value of RMSE gives the best result. The PSNR value is evaluated between the output de-noised speech signal and input noise mixed signal to verify the performance of the speech de-noising system. The system efficiency was tested by taking into consideration various speech samples. The parameters of the network structures used in the experiment is outlined in Table 1.

A set of trails are carried out to fix the neuron size of the layers of the networks. The iterative process varies depending upon various learning rates and different values of momentum. Table 2 summarizes the average test results obtained by different network structures. The RMSE values and PSNR values with variations in AWGN is summarized in the table. From Table 2. it is observed that SAE has got higher value of PSNR as compared to FFNN and RNN. The corresponding RMSE value is also shown. The best result is obtained for lesser value of RMSE and higher value of PSNR.

From Figure 6 it is observed that, in $-3 \mathrm{db}$ addition of noise, the SAE shows a PSNR value of 35.4 which is better than that of RNN ans FFNN models. With voice samples corrupted by $-1 \mathrm{~dB}$ SNR, the SAE model shows a performance improvement of around $5-12 \%$ that that of RNN and FFNN respectively. The significance of the result is that the degradation of the voice quality 
has no effect and the performance of the SAE is found to be nearly consistent. The experiment shows that the de-noising quality of SAE is better than that of the other learning based classifiers although the training latency of SAE is higher.

\begin{tabular}{|c|c|c|c|}
\hline Sl No. & Network & items & parameters \\
\hline 1 & FFNN & $\begin{array}{l}\text { Hidden layers } \\
\text { Training algorithm } \\
\text { Maximum epochs } \\
\text { Training type } \\
\text { Goal }\end{array}$ & $\begin{array}{l}\text { Two (with } 80 \text { and } 90 \\
\text { neurons respectively) } \\
\text { Scaled Conjugate } \\
\text { Gradient } 2000 \\
\text { Back propagation } 10^{-3}\end{array}$ \\
\hline 2 & RNN & $\begin{array}{c}\text { Hidden layers } \\
\text { Training algorithm } \\
\text { Maximum epochs } \\
\text { Training type } \\
\text { Goal }\end{array}$ & $\begin{array}{c}\text { Two (with } 40 \text { and } 50 \\
\text { neurons respectively) } \\
\text { Scaled Conjugate Gradient } \\
2000 \\
\text { Back propagation } 10^{-3}\end{array}$ \\
\hline 3 & SAE & $\begin{array}{l}\text { Maximum epochs } \\
\text { Loss function } \\
\text { Transfer function } \\
\text { Training algorithm } \\
\text { Weight regularization }\end{array}$ & $\begin{array}{c}5000 \\
\text { MSE } \\
\text { Purelin } \\
\text { Scaled Conjugate Gradient } \\
0.001\end{array}$ \\
\hline
\end{tabular}

Table 2. Performance measure of various network architectures.

\begin{tabular}{|c|c|c|c|}
\hline Network type & $A W G N$ in $d B$ & RMSE & PSNR \\
\hline \multirow[t]{6}{*}{ FFNN } & -3 & .21 & 22.1 \\
\hline & -2 & .20 & 25.3 \\
\hline & -1 & .18 & 26.8 \\
\hline & 1 & .16 & 28.4 \\
\hline & 2 & .14 & 31.5 \\
\hline & 3 & .11 & 32.6 \\
\hline \multirow[t]{6}{*}{ RNN } & -3 & .11 & 32.8 \\
\hline & -2 & .10 & 33.1 \\
\hline & -1 & .09 & 34.2 \\
\hline & 1 & .09 & 35.3 \\
\hline & 2 & .08 & 36.5 \\
\hline & 3 & .07 & 38.4 \\
\hline \multirow[t]{6}{*}{ SAE } & -3 & .09 & 35.4 \\
\hline & -2 & .08 & 36.8 \\
\hline & -1 & .07 & 39.4 \\
\hline & 1 & .06 & 41.2 \\
\hline & 2 & .05 & 42.6 \\
\hline & 3 & .04 & 44.5 \\
\hline
\end{tabular}

\section{CONCLUSION}

In this paper, we have focused on the design of an Autoencoder based de-noising of Assamese speech samples. Several speech samples are mixed with a range of Gaussian noises which introduces a range of de-noising conditions with variation in the noisy samples. From the experimental results it has been found that the SAE based approach yields a better result for de-noising purpose
Figure 6: Noise vs PSNR for SAE, RNN and FFNN

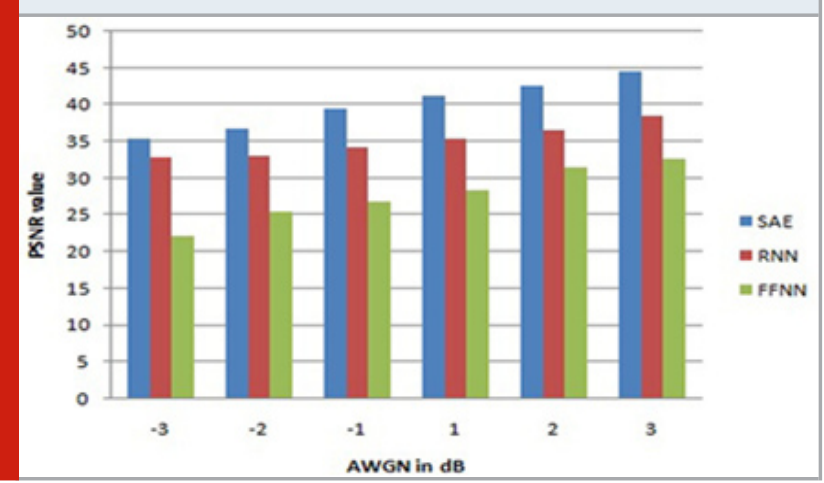

than other network types. The PSNR values obtained using SAE is better as compared to other models used for de-noising the speech samples. There is an improvement of around $6 \%$ to $11 \%$ in the PSNR value because of the use of SAE model. Despite of higher computational latency the proposed approach provides better signal de-noising and can be effectively used in real situations to remove various noises present in the signal.

\section{REFERENCES}

Baldi, P. 2012. Autoencoders, Unsupervised Learning and Deep Architectures. In proceedings of ICML Workshop on Unsupervised and Transfer Learning, 27: 37-50.

Badri, L. 2010. Development of neural networks for noise reduction. International Arab Journal of Information Technology, 7 (3): 289-294.

Cox , K. S. 1988. An Analysis Of Noise Reduction Using Back-Propagation Neural Networks. Thesis, Faculty of The School Of Engineering Of The Air Force Institute 
Of Technology, Air University, Master of Science In Computer Eng. Captain, Usaf, Afit/Gce/Eng/88d-3.

Daqrouq, K., Abu-Isbeih, I. N., \&t Alfauri, M. 2009. Speech signal enhancement using neural network and wavelet transform. In 2009 6th International MultiConference on Systems, Signals and Devices, 1-6, IEEE.

Feizi, A. 2019. Convolutional Gating Network for Object Tracking. International Journal of Engineering, 32 (7): 931-939, DOI: 10.5829/ije.2019.32.07a.05.

Mikolov, T., Karafiat, M., Burget, L., Cernock'y J., \&t Khudanpur, S. 2010. Recurrent Neural Network Based Language Model. In Eleventh Annual Conference of the International Speech Communication Association, 1045-1048.

Osako, K., Singh, R. \&t Raj, B. 2015. Complex recurrent neural networks for denoising speech signals. In 2015 IEEE workshop on applications of signal processing to audio and acoustics (WASPAA), 1-5, IEEE, DOI: 10.1109/ WASPAA.2015.7336896

Rajini, G. K., Harikrishnan, V., M., J. P. Priyadarisini Et Balaji, S. 2019. A Research on Different Filtering Techniques and Neural Networks Methods for Denoising
Speech Signals. International Journal of Innovative Technology and Exploring Engineering, 8 (9S2).

Svozil, D., Kvasnicka, V., \&t Pospichal, J. 1997. Introduction to Multi-layer Feed-forward Neural Networks. Chemometrics and Intelligent Laboratory Systems, 39(1): 43-62.

Sharma, M., Sarma, K. K., \&t Mastorakis, N. 2019. Aircraft image de-noising and identification using deep neural network. Int. J. Circuits Syst. Signal Process., 13: 430437.

Tamura, S. 1989. An analysis of a noise reduction neural network. International Conference on Acoustics, Speech, and Signal Processing. 3, 2001-2004, DOI: 10.1109/ ICASSP.1989.266851

Upadhyay, N. and Karmakar, A. 2013. An improved multi-band spectral subtraction algorithm for enhancing speech in various noise environments. Procedia Engineering, 64: 312-321. DOI: 10.1016/ j.proeng.2013.09.103

Wan, E. A., Nelson, A. T., \&t Katagiri, S. 1999. Networks for speech enhancement. Handbook of neural networks for speech processing. Artech House, Boston, USA, 139 (1): 7 . 